\title{
Integrated Reasoning Support in System Design: Design \\ Derivation and Theorem Proving
}

Steven D. Johnson

Indiana University Computer Science Department

Lindley Hall, Bloomington, IN 47405-4101, U.S.A.

1-812-855-2567 (tel), -4829 (fax)

sjohnson@cs.indiana.edu

Paul S. Miner

NASA Formal Methods Team

Langley Research Center, Hampton, VA 23681-0001

1-757-864-6201 (tel), -4234 (fax)

p.s.miner@larc.nasa.gov

Abstract
Practical applications of formal methods research require the integrated use of distinct tools for reasoning and design. Many approaches to this problem involve embedding specialized verification procedures in a theorem prover or logical framework. In fact, some theorem provers are promoted as frameworks of just this kind. We discuss some of the problems inherent to such monolithic treatments, illustrating with studies we have done in ad hoc heterogeneous reasoning. For both technical and pragmatic reasons we conclude that shallow embedding, that is, integration through superficial syntax translation, is a reasonable and even necessary approach.

\section{Keywords}

Formal methods, design derivation, theorem proving, hardware verification

\section{INTRODUCTION}

The impetus to write this paper was an article by Kaufmann, Good, and Moore (1992a) explaining their perspective on integrated formal system design. This perspective is summarized in the abstract: 
... the popular approach to imposing formal semantics on computational models, namely the creation of "formula generators," fails to formalize the very ideas allegedly being studied. In our view, this explains why so much work on system verification is thwarted by "integration" problems. These "integration" problems simply disappear when the ideas being studied are formalized and available for logical manipulation by the automated reasoning system.

While we find a lot to agree with in the ensuing article, we take virtually the opposite view of integration. We nevertheless allege that we are addressing substantive issues, even if our approach is the antithesis of what these authors espouse. There may be terminology problems involving notions of "formalization" and "formality" and their relation to mathematical rigor. A recent counterpoint by Guttman and Wand (1995) argues the case for rigor. However, from either perspective, it is clearly important to develop toward heterogeneous reasoning environments in which different styles of argument can be harmoniously used in specification, design and verification tasks.

Kaufmann, Good \& Moore assert that true path to successful integration lies in a common logic. Proclaiming "Logic Comes First," they invoke the names of The Ancients (Frege, Skolem, Church, etc.) for having done the science from which little remains to do but the engineering. This strident (and occasionally smug) rhetoric has at least the virtue of taking a firm stand; and it is backed by an impressive body of achievement.

We remain steadfastly ambivalent on these issues but will take the opposing stand for the sake of argument. The first century of mathematical logic has failed to achieve its intended goal of formalizing mathematical argument. See Allwein \& Barwise (1996), for example, or just observe that logic remains an active discipline. Furthermore, it is doubtful that the pioneers foresaw either the character of computer involvement in reasoning applications, or the demands that formalized programming imposes on model theory (See, for example, Aczel 1988).

The next century of logic would do well to contemplate engineering processes rather than mathematical proofs. We contend that no existing logic or logical framework is capable of a balanced treatment of reasoning styles (See Section 5, for example), much less of encompassing both logic and meta logic. Giunchiglia, Pecchiari \& Talcott (1994) survey this topic and introduce a formal approach to the metalogical framework.

The work described here focuses on the pragmatic aspects of integration. No existing mechanized logic is robust enough to host the software engineering necessary to produce and maintain an useful tool set. For an example of research focused on formal aspects of integration, the work on $T-R u b y / R u b y Z F$ by Sharp and Rasmussen (1997) is perhaps closest to ours in terms of the styles of reasoning involved. The relational algebra T-Ruby is similar to the derivational arguments we are concerned with. RubyZF, developed in the Isabelle framework, uses inference to verify conditions generated by T-Ruby transformations. 
We suggest that: Logic Comes Last! It is a way to consolidate engineering experience and - to the extent that this is successful-teach methods and implement supporting tools. The need to test prospective formalisms in realistic contexts motivates the exploratory work described in this article. We review three studies, each of which attempted coordinated use of two or more interactive tools in a single design context. One of these tools was always DDD, our own system for formalized design by derivation. We explored integrating DDD with three inference oriented reasoning tools: the Infer type system for Scheme (Haynes 1993), the Nqthm reasoning system (Boyer \& Moore 1988), and the $P V S$ verification system (SRI 1997).

Although we believe our experiences contribute to advances in applied research in integrated reasoning, we have drawn few firm conclusions from our studies, other than a justification for "shallow" interactions between formal systems. Generally, theoretical issues seem less challenging and even less urgent than software engineering problems, given the modest resources available to make formal methods research practical. Section 6 outlines some of the ideas we plan to explore next.

\section{INTEGRATION STUDIES WITH DDD}

DDD (Digital Design Deriver) is an interactive system of transformations and constructions for reducing system specifications to implementations (Bose 1994, Bose 1991). It operates on recursive systems of functions, representing first-order state transition systems, and recursive systems of streams, representing system architectures.

Figure 1 shows examples of the two forms. They are the canonical finite state machine and circuit expressions, given in terms of next-state and output functions, NS and OUT, respectively.

Figure 1(a) is a control oriented form. The recursive variables represent states of a transition system; this example has just one state, FSM. The formal parameters I, S, and 0 stand for for input, internal data state, and output, respectively. A state transition involves advancing the input history, updating the state, and performing an output action. The uninterpreted hd, tl, and append operations suggests a model of $\mathrm{I} / \mathrm{O}$ as list access operations. Condition ( $P$ I S) leads to an unspecified branch ( $T$ I' $S 0$ ). For this article, assume $T$ denotes termination, $(T I, S 0) \Rightarrow 0$.

Figure 1(b) illustrates an architecture oriented DDD expression. The recursive variables denote nonterminating sequences, represented in Scheme by streams. Sequence $S$ is the possibly infinite trace of internal states and 0 is its corresponding output history. In this form, operation symbols NS and OUT are reinterpreted as mappings over streams of their discrete counterparts in Figure 1(a). Scheme's language extension facility is used to make this expression executable. System-letrec is a macro that converts terms within to stream expressions. 


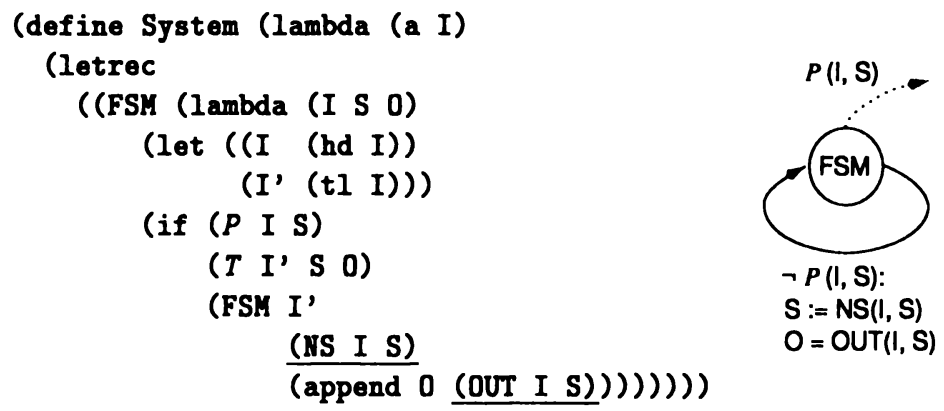

(FSM I a nil))))

(a) Control oriented expression

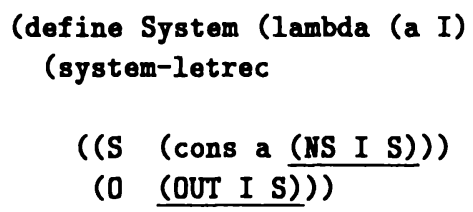

0)))

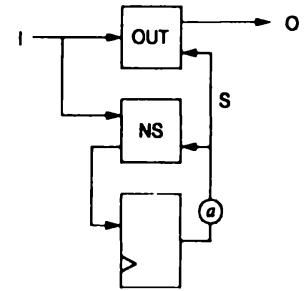

(b) Architecture oriented expression

Figure 1 DDD expression forms

DDD is a transformation system that operates on expressions of these forms. It as a first-order reasoning tool in which implementation proofs are presented as algebraic derivations. It is proficient at the large-scale formal manipulations involved as structure is imposed on a behavioral specification and as concrete data representations are introduced. A proof consists of an initial expression and a sequence of constructions and transformations together with any side conditions they generate. In practice, one also needs the intermediate expressions in order to address the subexpressions to be manipulated.

In developing DDD, our primary interest is investigating how a creative intelligence interacts with a computer at higher levels of design. Constructing a proof in DDD is an editing process that "adds information" to the specification while preserving behavioral equivalence. Such a system is bound to evolve a complicated graphical interface. Figure 2 shows the user's view of a recently released derivation tool called DRS (Derivation Systems, Inc. 1995), a successor to DDD developed after these studies took place. The snapshot contains windows for visualizing the expression being manipulated, managing the derivation process, performing animation and other analyses.

The textual content of Figure 2 is not important to this article, but the figure does indicate the considerable amount of software devoted to graphics and other support functions. This proportion will only increase as we work 


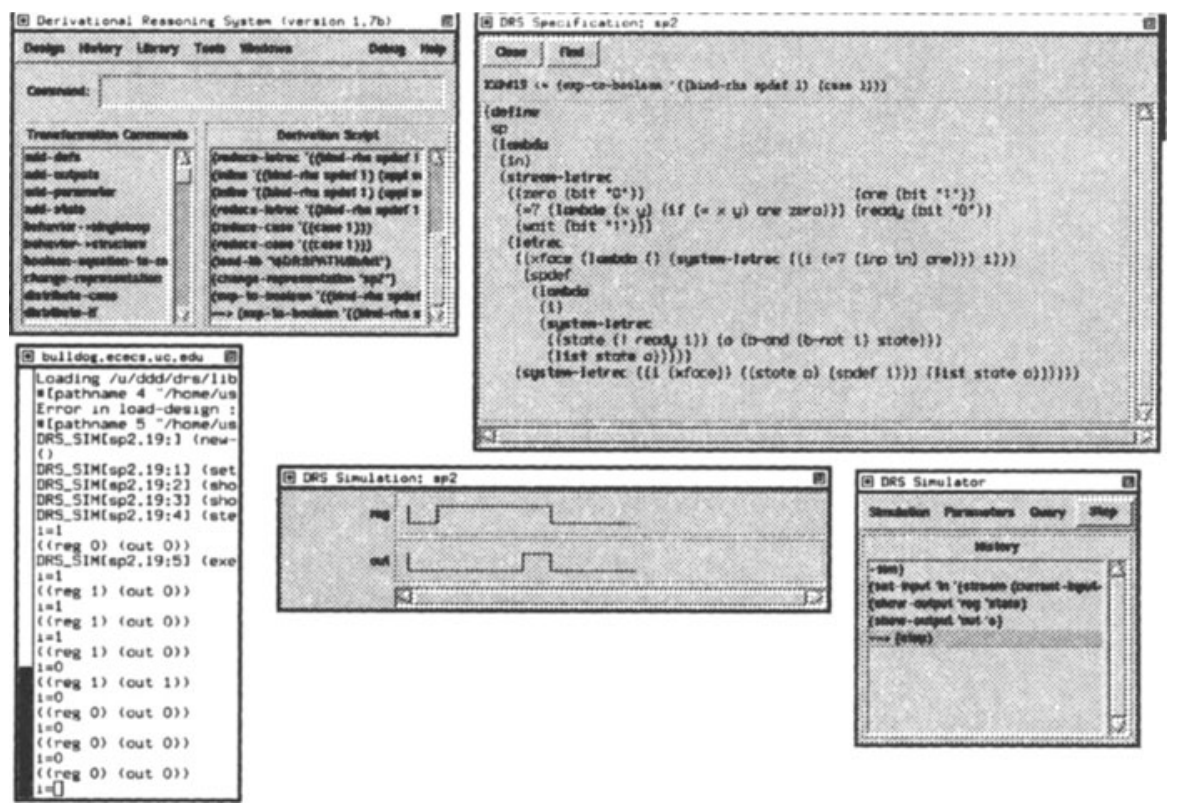

From the upper left, clockwise, are the derivation management window, containing a command menu, derivation history, and current command editing subwindow; the current expression window, used to address subexpressions; a control window for animating the model; a window showing signal wave forms; and a window for input to the model.

Figure 2 Snapshot of a DRS session

toward making design derivation more practical. The intensively interactive character of design derivation is a primary roadblock to its integration with other systems.

\section{INFER AND DDD}

Design derivation research began as an outgrowth of language and programming methodology work centering on the Scheme programming language. Scheme, a dialect of Lisp, is a latently typed language with mathematical foundations rooted in domain theory. For this reason, a type system was not integral to the original DDD algebra.

The conceptual need for abstract data types emerged quickly in our experimentation with DDD. We looked first to Haynes's Infer system (Haynes 1993) as potentially supporting types in way that was compatible with contemporary programming methods research based in Scheme. Infer is an ML-style type inference system superimposed on the Scheme user level. It supports, 


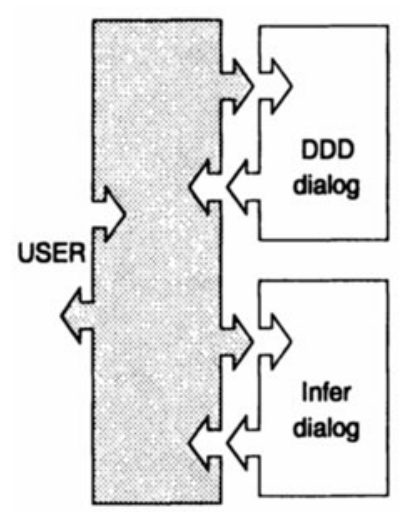

(a)

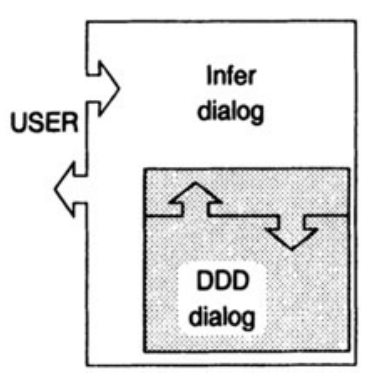

(b)

Figure 3 Two possibilities for integrating DDD and Infer

in a restricted way, the Scheme language extension facilities used in DDD to introduce stream semantics (Recall Figure 1(b)).

We also believed - as turned out to be the case - that integrating with Infer would teach us about future integration with a theorem prover. Infer's fundamental reasoning mode is inference, it maintains a knowledge base of theorems in the form of type assertions. Thus, one of the ground rules in our attempt at integration was that Infer should remain a fixed software component, since we could not expect an independently developed theorem prover to be altered for integration with DDD.

Like DDD, Infer encloses Scheme in an interactive shell. The example below is taken from Haynes (1993); user input is italicized.

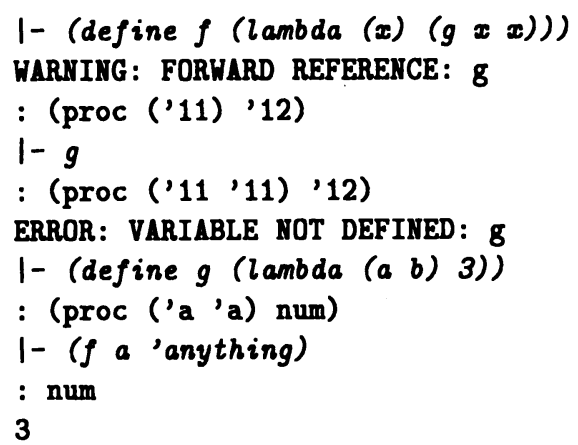

With no provisions for third-party interactions, implementing a collaboration among user, DDD, and Infer required either a highly interactive metasystem or a complete embedding of DDD within Infer. Although neither of the alternatives is attractive, building an arbiter interface with the user on 
one side and with DDD and Infer working independently on the other (Fig. $3(a))$ is, in retrospect, the more practical solution.

Since we wanted a more tightly coupled system, and had elected not to modify Infer, we embarked on a re-implementation of DDD within Infer (Fig. $3(b))$. There were three major roadblocks in the way of this approach, roughly, semiotic, linguistic, and metalinguistic in character.

DDD manipulates executable Scheme modeling expressions, and part of its functionality is to animate designs. To perform this animation within Infer, it was necessary to type not only the expressions manipulated, but their underlying semantics when it involved Scheme syntax extension. A stream implementation in Scheme involves continuations, threading, and assignment; it is not a trivial exercise in building types. The tangential development work dominated the the study, and deferred us from addressing the interesting issues.

Implementing DDD transformations in Infer entailed a formalization of syntax with implied updates to all existing code. DDD is not a huge piece of software, but the necessary work would have consumed a yet another considerable portion of our resources, had we undertaken it, which we did not.

Eventually, we realized (and should have recognized at the outset) that while implementation of DDD within Infer was possible, interaction between an internalized DDD and Infer was not. The Hindley-Milner type system on which Infer is based does not allow quantification over types. Thus, programs that query (i.e., construct queries) the underlying knowledge base, or access inference primitives such as the occurs check, or assert the type of an expression, are disallowed. So are programs that construct definitions.

As the fragment above shows, both nongeneric (e.g., ' 11 ') and generic (e.g., ' ' $a$ ') type symbols are generated by the Infer dialog. Thus, greater flexibility for metalinguistic reasoning about types is possible via the interactive dialog. This is why we concluded that the better alternative would be to develop a metasystem to mediate between DDD and Infer, as suggested by Figure 3(a). At this point we discontinued the study.

\section{NQTHM AND DDD}

When DDD was first developed, we looked for ways to contrast its algebraic methods with other treatments of system verification. An obvious candidate for us was Hunt's FM8501 proof, using the Nqthm theorem prover (Johnson, Wehrmeister \& Bose 1989). Bose carried this case study through (Bose \& Johnson 1993, Bose 1994) to the FM9001, a verified microprocessor realized in gate-array technology (Hunt, Jr. \& Brock 1997).

Both DDD and Nqthm operate on similar dialects of Lisp-close enough that we could apply DDD almost (see below) directly to Hunt's top level specifications. In other words, Nqthm was our best opportunity to integrate 
with theorem proving through a shallow syntax embedding. Bevier, et. al. (1989) give this schema for specifying machine behavior.

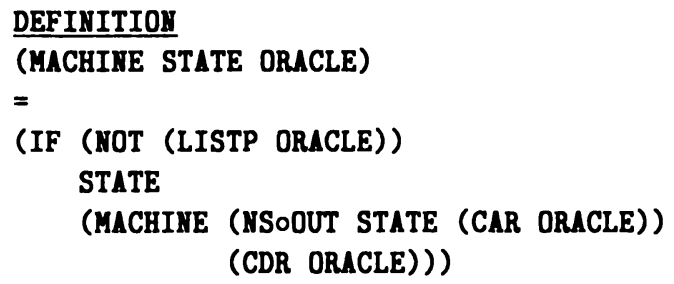

As suggested in Figure 1(a), The ORACLE parameter represents external events, inputs, as a list. Output is subsumed in the STATE object, in effect composing the NS and OUT functions of the figure.

There were two significant semantical problems in coordinating DDD and Nqthm modeling expressions. Both systems use tail-recursive functions to express state-transition models. Hunt uses a proper hierarchy of six functions to describe FM9001 at the programmer's instruction level, plus an iteration theorem to model execution of instruction sequences. DDD uses a mutually recursive system of functions to describe cyclic transition systems. The technique for dealing with simultaneous recursion in Nqthm involves translation to a single recursion with encoded function names. It is very cumbersome to keep track of this encoding while performing transformations on control oriented forms.

The same problem is compounded in architectural descriptions, where DDD uses recursive networks of streams to model synchronous hardware. Animation of streams in Scheme is done using function closures and continuations to implement call-by-need behavior. Neither of these higher-order objects can be expressed directly in Nqthm, nor can nonterminating function systems.

Hunt overcame all these problems by a deep embedding, defining a structural hardware description language, DUAL-EVAL, and its interpretation in the logic (Hunt, Jr. \& Brock 1997). He reports that these definitions-all of which are reusable - constitute over a third of the FM9001 proof statement. The DUAL-EVAL counterpart to Figure 1(b) would be:

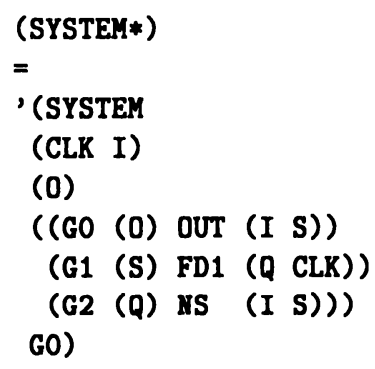

Were we to implement DDD in Nqthm (now ACL2), we would probably follow Hunt's lead and use a deeply embedded syntax, such as this. However, 
DUAL-EVAL is grounded in binary, and it would be a matter for further research to formalize a first-order variant. In addition, we would probably be compelled, also, to embed the DDD's control oriented dialect, since these expressions are subject to transformation. One problem with doing this is that we loose access to the logic as a specification language: we cannot assert from within Nqthm that if embedded expression $E$ satisfies logical property $F$, then constructed specification (DDD $E$ ) also satisfies $F$. The quantification over $F$ is higher order.

Using DDD, Bose was able to derive gate networks from Hunt's FM specifications. In the process, he exploited large portions of Hunt's proof by incorporating the binary implementation of Hunt's ALU. Thus, while the DDDFM9001 implementation had a different data-path architecture, it shared large portions of the combinational arithmetic.

Hunt developed parameterized module generators using DUAL-EVAL producing and ALU description that was both correct and efficient for his LSI target technology. However, these implementations did not map well to the Actel FPGA technology we targeted for the DDD case study. Instead, the Nqthm functions specifying binary arithmetic were symbolically expanded to produce naive ALU specifications, against which we verified a manual design using extractors and an equivalence checker (Bose, Johnson \& Pullela 1993).

Bose's derivation resulted in hardware that compared directly at the instruction level to Hunt's LSI realization (Bose 1994). Beneath the instruction level granularity, the two devices were significantly different. A important difference lies in the memory implementations used. The FM specification models memory as a simple, functional object, subject to atomic read and write operations. In Hunt's implementations, memory transactions involve an explicit, four-phase handshake.

Hunt dealt with this refinement by modifying his correctness theorem, using the ORACLE parameter to represent asynchronous memory behavior. At the time of this study, the DDD algebra could not handle such refinements to timing and synchronization. Had a synchronization handshake simply been added to the formally derived implementation, a correctness proof would have been required to validate the newly imposed memory model. Although this kind of refinement has since been added to DDD (Rath, Choppella \& Johnson 1995, Rath 1995), it remains a good example of the potential need for heterogeneous reasoning in support of ad hoc refinements. It is difficult to contemplate a general interaction between DDD and Nqthm that would maintain correctness in cases like this.

As was the case with Infer, we have the alternatives of Figure 3 for integrating the two systems. Reimplementing DDD within Nqthm (or ACL2), while perhaps interesting to a formalist, would delay further advances in derivation methodology, inhibit user interface development, and, in all likelihood, raise order problems in the Boyer-Moore logic. From our perspective, it would be 
more expediant and more fruitful to create a metasystem to manage syntactic transactions between Nqthm and DDD.

\section{PVS AND DDD}

Miner is investigating the use of DDD and the PVS verification system (SRI 1997) for hardware system design. His case studies address both the practical issues of integration and the stratification of verification tasks (Miner \& Leathrum 1996, Miner \& Johnson 1996, Miner 1997). For example, his formalized design of a clock synchronization circuit involves the mathematical characterization of the synchronization problem (Miner 1993), the specification of a cyclic procedure and the design level proof that it meets the requirements, and derivation of a nontrivial hardware implementation. In this section, we discuss three pervading issues for integration.

\subsection{ADT translations}

DDD provides a collection of transformations to replace an abstract data type occurance with a more concrete representation. Incorporating a representation requires defining a correspondence between terms in the abstract ADT and combinators in the concrete ADT. DDD assumes that a given representation has been verified, that is, that an implementation relationship has been established between the two ADTs. We usually work with a simple notion of implementation, involving a partial equivalence relation on the (term algebra of) the concrete type which supports the equational theory of the abstract type.

More complicated notions of implementation are necessary, and are the subject of current research. For example, the implementation algebra will involve contingencies, such as overflow. Even more important are interface issues, such as the introduction of handshaking signals, as mentioned at the end of Section 4.

The clock synchronizer study illustrates several aspects of this problem. The design specification contained an abstract representation of clock mappings as ordered sets (lists, actually) of integers. These sets were initially translated to arrays (because the sets had bounded sizes) and subsequently a simple pair (because only two elements were ultimately needed) of integers in the circuit. At a much later stage in the implementation the pair of integers was replaced by a single counter, discussed in Section 5.4. With the possible exception of the last, these translations can all be formalized as representation refinements involving a correlation of ADTs.

Although PVS has ample provisions for ADT specification, its type system, like Infer's, disallows type variables. Hence, formalizing theorems about ADT implementation relationships would appear be outside the logic. We need 
something like type universes in Nuprl (Constable et al. 1986). Resolution of this problem, whether within the logic, or within the PVS preamble, or via a collection of proof strategy generators, is a matter best left to the PVS implementors, but it would be of considerable value to a system like DDD.

\subsection{Mutual recursion, again}

As was the case with Nqthm, any embedding of DDD expressions is hindered by the fact that neither simultaneous recursion nor nontermination is allowed in function definitions. Higher order types open additional avenues for attacking this problem, but any such attack imposes a layer of interpretation to be maintained either within the proof management system, or in the user's mind.

\subsection{Streams}

In DDD, systems are modeled as networks of streams, which are infinite sequences over a type. Streams and other nonfinite structures raise issues in model theory that are only now being ironed out (Barwise \& Moss 1996). These problems are intrinsic to logics based in well-founded set theory, which must deal with streams indirectly. The typical representation is mapping from natural numbers to values. The problem is that proofs about streams under this representation reduce to inductions over the naturals, instead of "structural" coinductions that reflecting the definition style.

This problem is greatly compounded by the lack of intrinsic support for simultaneous recursion, which is essential in network descriptions. The user is now contending with two levels of interpretation, resulting in an overwhelming amount of bookkeeping in the course of a routine argument.

Miner axiomatizes a parameterized type, Stream $[\alpha]$ with cons, head, and tail constructors subject to the usual identities. He defines an indexing accessor, nth: (Stream $[\alpha]$, Nat) $\rightarrow \alpha$ and then postulates:

$$
S_{1}=S_{2} \quad \text { iff } \quad\left(\forall n: \operatorname{nth}\left(S_{1}, n\right)=\operatorname{nth}\left(S_{2}, n\right)\right)
$$

With these foundations, the goal is to establish definition and proof schemes that are compatible with DDD network manipulations. A canonical streamdefinition scheme is:

$\operatorname{corec}(f, g)(a) \triangleq \operatorname{cons}(f(a), \operatorname{corec}(f, g)(g(a)))$

This scheme, together with an extensionality theorem stating that bisimilar streams are equal, form the basis of a PVS proof strategy that automates routine assertions about streams and predicates over them. 
An embedding of DDD stream networks relies on PVS's dependent-type capabilities (Miner \& Johnson 1996) and does the "plumbing" needed to accommodate simultaneous recursions. Figure 1(b) is built in PVS as

$$
\begin{aligned}
& \text { system(out, ns) }(I, s):\{O \mid O=\operatorname{corec}(\lambda I s . o u t(I, s),(\lambda I s . n s(I, s))(I, s)\} \\
& \text { state(ns) }(I, s):\{S \mid S=\operatorname{corec}(\lambda I s . s,(\lambda I s . t I(I), \operatorname{ns}(\operatorname{hd}(I), s))(I, s)\}
\end{aligned}
$$

Together with a plumbing theorem

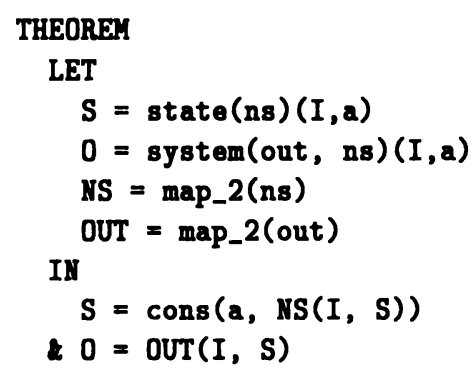

This predicate establishes a logical context for deductive reasoning about the components of the network in Figure 1(b).

\subsection{Conditional transformations}

From the DDD side, interactions with PVS are usually initiated by replacement transformations, that is, variants of the rule

$$
\frac{\Delta \vdash Y=F[x / T] \quad, \quad C \vDash R \equiv T}{\Delta, C \vdash Y=F[x / R]}
$$

in which there are two modes of inference: one from DDD $(\vdash)$ and the other from an external logic $(\models)$, PVS in this case. In exploring this interplay we have encountered a number of complications to be addressed in a more rigorous integration of PVS and DDD. These are probably familiar to anyone doing formal system design.

Typically, "replacement" refers to subsystems, rather than subterms, and so are sensitive not only to variable clashes but to the context of the surrounding system. Contextual information must be exported to support localized arguments about equivalence.

Equivalence itself (' $\equiv$ ') is contingent, with different notions of equivalence associated with different classes of transformations. For example, in his clock synchronization study, Miner replaces a pair of registers which are averaged, with a more clever counter, which skips clock cycles. These are subsystems consisting of several equations each, between which, fortunately, a strong 
bisimulation can be established at the finer time scale. We would like to customize the reasoning environment to subsume even weaker forms of equivalence under more complex temporal mappings.

As discussed earlier, the disposition of constraints (' $C$ ') is a design management problem. A peerhood relationship between PVS and DDD must include a framework for enforcing conditions when appropriate. In the counter optimization just described, bisimulation is contingent on certain input behaviors, hence these conditions are inherited as constraints on any system employing the synchronization circuit.

An example of the interplay arises in the following derivation fragment, taken from (Miner 1997, Ch. 6). It is a local, tactical transformation on the stream equations

$$
\begin{aligned}
& Q=\operatorname{reg}(?, \operatorname{sel}(C, 0, f(P, Q, R))) \\
& R=Q-1
\end{aligned}
$$

Reg is a register, sel is a selector, and ? is a don't-care. Recall that operators are extended to mappings; for instance, the term $Q-1$ denotes mapping subtraction over $Q$ and an infinite sequence of 1s. Expanding $Q$ in the defining expression for $R$, with some retiming and distribution of -1 through sel, we can write

$$
\begin{aligned}
& Q=\operatorname{reg}(?, \operatorname{sel}(C, 0, f(P, Q, R))) \\
& R=\operatorname{reg}((?-1), \operatorname{sel}(C,(-1), f(P, Q, R)-1))
\end{aligned}
$$

Now a property of $f$ is that $f(P, Q, Q-1)-1=g(P, Q, Q-1)$, which justifies replacing $R$ 's defining expression with

$$
\begin{aligned}
& Q=\operatorname{reg}(?, \operatorname{sel}(C, 0, f(P, Q, R) \\
& R=\operatorname{reg}((?-1), \operatorname{sel}(C,(-1), g(P, Q, R)))
\end{aligned}
$$

However, verifying this transformation depends on the fact that $R=Q-$ 1 , which can be also be read as the temporal assertion "Signal $R$ always contains the value on signal $Q$ minus 1 ." Unfortunately, this fact was, in effect, forgotten after the first transformation. Thus, a mechanism is needed to carry the information forward, for later use.

\section{COMMENTS AND RECOMMENDATIONS}

The important, and deeper, integration problem facing formal methods research involves the interactions of a creative, ingenious designer with the tools of design. The nature of this interaction is elusive because good designers adapt to the limitations of their tools much more quickly than the 
tools can be enhanced to accommodate the designer's techniques. Notions of expressiveness and completeness notwithstanding, we believe it is more urgent to explore disparate modes of reasoning with a view toward creating useful tool sets. Though we should all work toward a well integrated, uniform environment, we have het to learn how individual tools are used, both alone and in concert with other tools.

Integrating autonomous reasoning systems is neither a matter of formula generation (i.e. casting theorems of one as theorems of the other) nor of embedding (i.e. representing one system as a theory of the other). There must also be a correlation of proof processes because formalized "design" is the construction of a proof, not the final proof artifact. Where one of the components is automatic, the correlation can be made transparent, as, for example the embedding of linear arithmetic in Nqthm (Kaufmann, Good \& Moore 1992a). However, this approach does not work, or at least is highly difficult, when both tools are interactive. Our studies suggest to us that integration should be outside both systems, for several reasons.

- None of the systems involved had adequate facilities for user interface development. This alone precludes a full embedding of one system inside one of its peers, even if that were desirable.

- All of the systems assumed interaction with a human user, and offered no channel (other than files) for third-party input.

- A true need for metalogical reasoning arose in each of our studies. In the Infer and PVS studies, type sensitive DDD transformations require access to the underlying type system. Programming such access introduces type variables. Although we did not confront typing in the Nqthm study, a bidirectional interaction between Nqthm and DDD would have involved the construction and assertion of definitions, which would need to be routed through the user interface.

If our attempt to use Infer to provide a type inference system for DDD had been successful, interactions PVS would have been even more convoluted. What we would like, of course, is to share the same inference system. For that matter, we would also like software access to other facilities, like the term rewriter.

- PVS and Nqthm allow only simple forms of recursion involving a single recursive symbol. It may be possible to build front ends to these systems to handle recursive systems, but we did not have the expertise to do it.

- Streams (and other nonfinite or nonterminating objects) are very natural for modeling systems. All of the inference systems had problems representing streams. To some extent, DDD has this problem too: stream networks are a syntax extension and, therefore, a shallow embedding in the Scheme "theory."

These are all instances of the "integration' problems" dismissed as method- 
ological failures in Kaufmann et al. (1992a). We should instead, according to their perspective, incorporate Infer in DDD, and then perhaps DDD in ACL2, as nested "open black boxes." Even if this paradigm makes sense formally, and this is questionable, implementing embeddings consumes effort that could otherwise be devoted to more illuminating investigations.

Although the semantics of metalogical frameworks is certainly of interest, their pragmatics should take precedence in applied research. A coherent reasoning environment should reflect experience. The DRS system for design derivation, mentioned in Section 2, Figure 2, addresses some of the problems encountered in these studies. It contains a primitive type system to support type sensitive transformations. It provides parameterized component generation compatible with that used by Hunt for the FM9001. It maintains a file of correctness conditions generated by replacement transformations. It has "formula generators" to translate stream networks to co-rec definitions targeted to Miner's PVS theory of streams. These provisions are not necessarily solutions to underlying problems, but they do enable further investigations of heterogeneous reasoning.

In applied formal methods research, more work is needed at the foundations for software integration. Hence, many of our recommendations are softwarc engineering truisms.

The implementors of interactive reasoning tools should look for common ground in user interface development. Not only would this ease the task of building heterogeneous systems, but it might also simplify learning the systems.

The tools should be designed with multiparty interaction in mind, particularly communication channels to other software objects. Since several implementation languages are involved, it is likely that most the communication will be textual, and machine oriented interchange languages are helpful in this regard.

Subsystems should evolve toward sharable and reusable objects. The example pervading our studies our unsuccessful attempts to incorporate a type inference system for DDD instead of implementing one afresh.

There must also, of course, be more research into formal aspects. This research should not ignore the ad hoc frameworks emerging in applications, that is, the cut-and-paste operations done by those attempting to manually integrate these tools in practice.

\section{ACKNOWLEDGMENTS}

Much of the work described was done with support from the National Science Foundation, under grants MIP-8921842 and MIP-9208745. Jean-Yves Marion contributed in many ways to the formulation of a coinductive theory of streams. Bhaskar Bose, M. Esen Tuna, Venkatesh Choppella, Shyamsundar Pullella, and Willie Hunt participated in the experimentation. The system 
shown in figure 2 was developed at Derivation Systems, Inc., of Carlsbad California, based on the research system we call DDD.

\section{REFERENCES}

Aczel, P. (1988), Non-Well-Founded Sets, CLSI Lecture Notes No. 14, CLSI Publications, Stanford.

Allwein, G. \& Barwise, J., eds (1996), Logical Reasoning with Diagrams, Oxford University Press.

Barwise, J. \& Moss, L. (1996), Vicious Circles, CLSI Lecture Notes No. 60, CLSI Publications, Stanford.

Bevier, W. R., Hunt, W. A., Moore, J. S. \& Young, W. D. (1989), 'An approach to systems verification', Journal of Automated Reasoning .

Bose, B. (1991), DDD - a transformation system for digital design derivation, Technical Report 331, Indiana University Computer Science Department.

Bose, B. (1994), DDD-FM9001: Derivation of a Verified Microprocessor, PhD thesis, Computer Science Department, Indiana University, USA. Technical Report No. 456, 155 pages.

*ftp://ftp.cs.indiana.edu/pub/techreports/TR456.ps.Z

Bose, B. \& Johnson, S. D. (1993), DDD-FM9001: Derivation of a verified microprocessor. an exercise in integrating verification with formal derivation, in G. Milne \& L. Pierre, eds, 'Proceedings of IFIP Conference on Correct Hardware Design and Verification Methods', Springer, LNCS 683, pp. 191-202.

Bose, B., Johnson, S. D. \& Pullela, S. (1993), Integrating boolean verification with formal derivation, in D. Agnew, L. Claesen \& R. Camposano, eds, 'Proceedings of IFIP Conference on Hardware Description Languages and their Applications', Elsevier, pp. 127-134.

Boyer, R. \& Moore, J. S. (1988), A Computational Logic Handbook, Academic Press, New York.

Constable, R. L. et al. (1986), Implementing Mathematics with the Nuprol Proof Development System, Prentice Hall.

Derivation Systems, Inc. (1995), DRS: Derivational Reasoning System, Version 1.2.1, Carlsbad, California. Contact drs@derivation.com.

Giunchiglia, F., Pecchiari, P. \& Talcott, C. (1994), Reasoning theories: Towards an architecture for open mechanized reasoning systems, Technical Report CS-TN-94-15, Stanford University Computer Science Department.

*Access via http://elib.stanford.edu/

Guttman, J. D. \& Wand, M., eds (1995), VLISP: A Verified Implementation of Scheme, Kluwer, Boston. Originally published as a special double issue of the journal Lisp and Symbolic Computation (Volume 8, Issue $1 / 2)$. 
Haynes, C. T. (1993), Infer: A statically typed dialect of scheme, Technical report, Indiana University Computer Science Department. Version 1.0. Limited distrubution draft, contact chaynes@cs.indiana.edu.

Hunt, Jr., W. A. \& Brock, B. C. (1997), 'The DUAL-EVAL hardware description language and its use in the formal specification and verification of the FM9001 microprocessor', Formal Methods in System Design. In press, contact hunt@cli.com.

Johnson, S. D., Wehrmeister, R. \& Bose, B. (1989), On the interplay of synthesis and verification: Experiments with the FM8501 processor description, in Claesen, ed., 'Applied Formal Methods for Correct VLSI Design', Elsevier, pp. 385-404.

Kaufmann, M., Good, D. I. \& Moore, J. (1992a), The role of automated reasoning in integrated system verification environments, in 'TTCP XTP-1 Workshop on Effective Use of Automated Reasoning Technology in System Development', Naval Research Laboratory, Washington DC. See also (Kaufmann, Good \& Moore 1992b).

Kaufmann, M., Good, D. I. \& Moore, J. (1992b), The role of automated reasoning in integrated system verification environments, Technical Report 73, Computational Logic, Inc., Austin TX.

*http://www.cli.com

Miner, P. S. (1993), Verification of fault-tolerant clock synchronization systems, NASA Technical Paper 3349, NASA Langley Research Center, Hampton, VA.

Miner, P. S. (1997), Hardware Verification using Coinductive Assertions, PhD thesis, Computer Science Department, Indiana University, USA. To appear.

Miner, P. S. \& Johnson, S. D. (1996), Verification of an optimized faulttolerant clock synchronization circuit, in M. Sheeran \& S. Singh, eds, 'Designing Correct Circuits', Electronic Workshops in Computing, Springer-Verlag.

${ }^{*}$ http://www.springer.co.uk/ewic/workshops/DCC96

Miner, P. S. \& Leathrum, Jr., J. F. (1996), Verification of IEEE compliant subtractive division algorithms, in M. Srivas \& A. Camilleri, eds, 'Formal Methods in Computer-Aided Design, FMCAD '96', Vol. 1166 of Lecture Notes in Computer Science, Springer-Verlag, Palo Alto, CA, pp. 64-78.

Rath, K. (1995), Sequential System Decomposition, PhD thesis, Computer Science Department, Indiana University, USA. Technical Report No. 457, 90 pages.

$*_{\mathrm{ftp}}$ //ftp.cs.indiana.edu/pub/techreports/TR457.ps.Z

Rath, K., Choppella, V. \& Johnson, S. D. (1995), 'Decomposition of sequential behavior using interface specification and complementation', VLSI Design Journal 3(3-4), 347-358.

Sharp, R. \& Rasmussen, O. (1997), 'The T-Ruby design system', Formal 
Methods in System Design . In press, contact osr@id.dtu.dk. SRI (1997), 'The PVS home page'.

*http://www.csl.sri.com/pvs.html

\section{BIOGRAPHY}

Steven D. Johnson is an associate professor of computer science in the Indiana University College of Arts and Sciences. He received a B.A. in mathematics and Russian from Depauw University in 1970, an M.A. in mathematics from Indiana University in 1977 , and the Ph.D. in computer science from Indiana University in 1983. His research is in the area of formal methods for system design. He is a member of the IEEE Computer Society, ACM, and IFIP WG 10.5 .

Paul Miner is a Research Engineer with the Formal Methods Team at NASA Langley Research Center. He will complete his Ph.D. in Computer Science from Indiana University in the fall of 1997. He holds a B.S. in Computer Science from Old Dominion University and an M.S. in Computer Science from the College of William and Mary. His research interests include application of formal techniques to digital hardware design and analysis of fault-tolerant systems. 